\title{
Research on Cleaning Intelligent Control System of Rice and Wheat Combine Harvester
}

\author{
Zhengxing Xiao ${ }^{1}$, Qing Jiang ${ }^{2,}{ }^{*}$, Zhengyong Zhang ${ }^{2}$, Rujin Wang ${ }^{2}$, \\ Liangtu Song ${ }^{2}$, He Huang ${ }^{2}$, Liusan Wang ${ }^{2}$ and Min Wang ${ }^{2}$ \\ 'Shenzhen Polytechnic, Shenzhen, China; \\ ${ }^{2}$ Institute of Intelligent Machines, Chinese Academy of Sciences, Hefei, China. \\ *jiangqing@iim.ac.cn
}

Keywords: Intelligent Control; Combine Harvester; Case-based reasoning; Expert System.

\begin{abstract}
Abstract: The intelligent regulation and control strategies for rice and wheat combine harvesters' operation are lacking and the rule of parameter matching is fuzzy in China, around these issues, the dynamic correlation control law among the parameters of rice and wheat, cleaning operation parameters of combine harvesters, the cleaning loss rate and trash content are studied, and so on. The intelligent control model for the rice and wheat combine harvester is established based on case-based reasoning, and achieve different rice-wheat breeds, moisture content and other rice-wheat properties, and provide clean fan speed, plate angle and scale screen control scheme with low trash content and cleaning loss rate. Through the development of web-based cleaning intelligent control expert system and conduct experimental evaluation, the feasibility and effectiveness of the case-based reasoning method in the intelligent control filed of rice and wheat combine harvesters is verified.
\end{abstract}

\section{Introduction}

China is a vast country, main grain production areas are widely distributed, and climatic conditions are different, therefore, there are many varieties of rice and wheat in main grain production areas, even if the grain-straw ratio or moisture content of homogeneous rice or wheat are also different [1,2]. In addition, through repeated experiment of field experiments and cleaning mechanical simulation platforms, many scholars have proved that the grain-straw ratio and moisture content of rice or wheat have important influence for the working parameters of the combine harvester cleaning and the related cleaning loss rate and trash content. The $[3,4,5,6]$. At present, the research on the cleaning control strategies of rice and wheat combine harvester mainly focus on the research of fuzzy control methods, there is still lack of intelligent control methods to reveal the correlation rule among ricewheat properties, cleaning operations and performance parameters. The similarity between casebased reasoning and fuzzy control is that there is no need to build an accurate mathematical model for the cleaning machine system. At the same time, it has a significant advantage over the fuzzy control method, it does not require artificial intelligence (AI) experts and field experts to spend too much effort to write a large number of rules, design membership functions, etc., but by building a historical case base and performing case retrieval, from which the historical cases with the greatest degree of similarity to the current case are selected, and finally the cases are reused to learn or adjust and modify the optimal historical case, thus obtaining the optimal solution of the current cases. Therefore, this research is aimed at the characteristics of rice-wheat varieties and attributes related to the cleaning operation and performance, utilizes cleaning intelligent cleaning system integrated in field test vehicle of rice and wheat combine harvester, accumulates historical case data in field experiments conducted in typical regions of China's main grain production areas, constructs cleaning control case base, and propose a case-based reasoning intelligent rice and wheat combine harvester model. It plays an important role in improving the operation efficiency of the main grain-production areas and reducing harvest losses, and it is of great significance to improve the level of China's agricultural mechanization. 


\section{Cleaning Intelligent Control System of Rice and Wheat Combine Harvester}

\subsection{Machine Drawing of Clearing Intelligent Control}

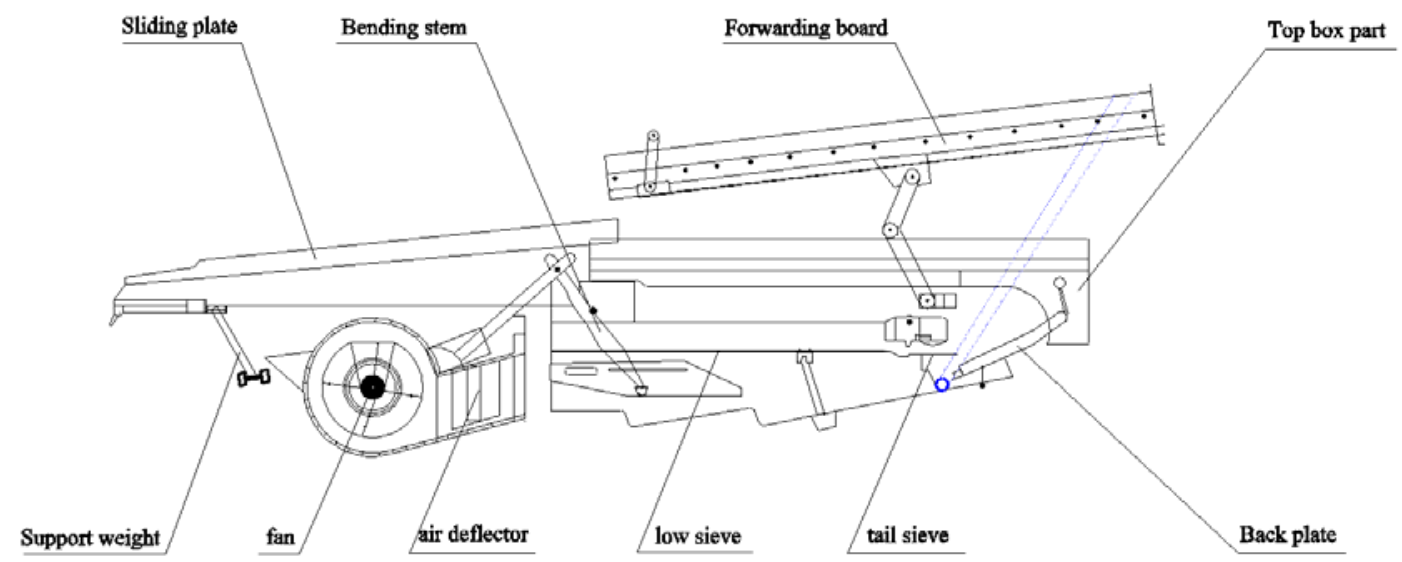

Figure 1. cleaning device of rice and wheat combined harvester

As shown in the above Figure 1, it is the structural diagram of the domestic mainstream $10-12 \mathrm{~kg}$ feed quantity rice and wheat combine harvester cleaning components, this cleaning mechanical system is mainly composed of sliding plate, bending stem, forwarding, top box part, support weight, fan, air deflector, low sieve, three tail sieves, back plate, etc.

During the operation, cleaning sieve and sliding plate both shuttle. The mixture in the flow cylinder falls on the sliding plate, and the mixture are sent to the back of the sliding plate by the shaking and conveying of sliding plate, under the cooperation of the cleaning fan and the fan, different materials are blown to different places.

Among them, lighter impurities and grains are blown up to the screen surface of the fish scales, and heavier grain and impurities are blown down to the screen surface of the fish scales. The materials on the upper sieve after the reciprocating motion, some grain will fall to the lower screen, light impurities will continue to blow to the tail and tail screen of the upper screen under the airflow action; The mixture of lower sieve under the reciprocating motion and airflow of the screen surface, grain falls on the grain skate plate, continue to slide towards the bottom of the grain elevator on the grain skate plate, deliver them to the grain tank through the transport of the elevator, other small amounts of the mixture continue blowing towards the end sieve under the effect of the air flow. In the tail sieve part, the impurities on upper sieve and lower sieve may have a small amount of grain or non-peeling seed heads, when these mixtures hit the back plate, drop onto the skate plate; finally, they are delivered to the sliding plate by impurity elevator for threshing. The glume and other impurities are directly blown out of the body and scattered in the field.

\subsection{Cleaning Intelligent Control and Actuator}

The structure of the cleaning mechanical control system is shown in Figure 2; it mainly includes the speed sensor, angle sensor, controller, electric push rod and computer. This system mainly completes the real-time monitoring and automatic control of fan speed, draft distributing plate angle, screen opening, loss rate, and trash content of rice and wheat combine harvester. The sensor system separately collects the value of each control quantity and sends it to the controller through the bus; the controller sends the data obtained to the host computer through the serial port communication. The host computer acquires and analyzes the data, enters the fuzzy computation system, sends the result of the fuzzy output to the controller, and drives the three electric push rods to drive their respective actuator so as to obtain the ideal cleaning effect. The whole system can realize the automatic operation of the rice and wheat combine harvester cleaning system with 10 to $12 \mathrm{~kg} / \mathrm{s}$ feed quantity, compared with the current manual operation mode, it not only can reduce the operator's labor, but also its cleaning effect is better than manual control effect. 


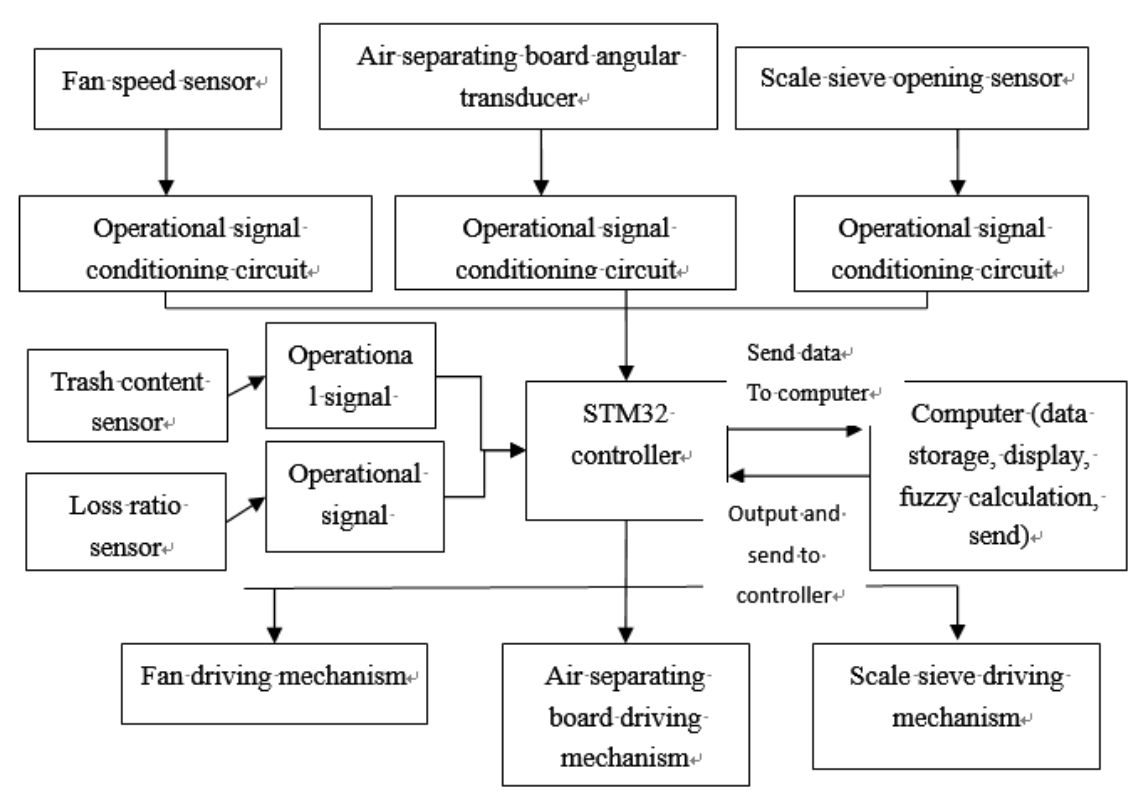

Figure 2. The structure of the monitoring system for cleaning

Due to its simple structure, the electric push rod is easy to install and take up less space. At the same time, it can support high thrust and high precision. The adjustment of the draft distributing plate and scale sieve opening in the system; its actuator is completely controlled by push rods. In the mechanical structure of the draft distributing plate and the scale sieve, a linkage rod is installed, respectively, and the linkage rod is connected with the push rod. When the expansion and contract command of the controller is transmitted to the push rod, the push rod will expand and contract, thereby driving the adjustment of the draft distributing plate angle and the scale sieve opening.

The adjustment of the fan speed is achieved by a combination of engine and push rod. First, the rotation of the engine drives the pulley, and promotes crank rocker of fan speed rotate, give an initial velocity to the fan. Adjustment of fan speed can be divided into coarse and fine adjustment; coarse adjustment is to change the fan speed by artificially changing the fan speed, fine control is to control the expansion of the push rod through the command of the controller, and then drive the rotation of the crank rocker to change the speed of the fan. Coordination between fine and coarse control, its control precision will be greatly improved.

Fan speed sensor is mainly responsible for collecting fan speed to calculate the main control unit. Sensors use Hall speed sensors; the output signal is a square wave signal, use the controller's timer to acquire the sensor's speed pulse signal, and can get the speed information. The normal working voltage of this sensor is 3 to $24 \mathrm{~V}$; the voltage provided on the vehicle is $24 \mathrm{~V}$ in the system, so the vehicle-mounted power supply can be directly used for power supply without voltage conversion.

According to the expansion length of the electric push rod and the size of draft distributing plate, draft distributing plate sensor establishes correspondence, the controller can get the angle of the draft distributing plate by obtaining the length of the electric push rod. The scale screen opening sensor is similar.

STM32 controller uses STM32F103ZET6 chip as the control core, which has 12 PWM power outputs, 2 switch quantity inputs, 6 analog quantity inputs, $824 \mathrm{~V}$ power outputs, 2 full-duplex serial ports, 1 CAN interface and so on.

The lower computer of the system adopts Keil software for programming, which mainly accomplishes the collection of data signals, communicate with the host computer and send the drive signal of actuator, the upper computer development uses the VS2010 as a development tool, mainly realizing the real-time monitoring, parameter storage, algorithm processing of relevant parameters of the cleaning system. STM32 real-time acquisition sensor send data to the host computer, the host computer will save the sensor data to the database. The upper computer runs cleaning intelligence model based on case-based reasoning, according to the crop varieties and rice-wheat property data, the cleaning operating condition parameter data is calculated, and the command signal is sent to the lower computer to drive the corresponding actuator. For the entire process, all the input and output 
data involved, we store and save the data in a certain format on the host computer, so that we can analyze the data of the system later. In Figure 3.

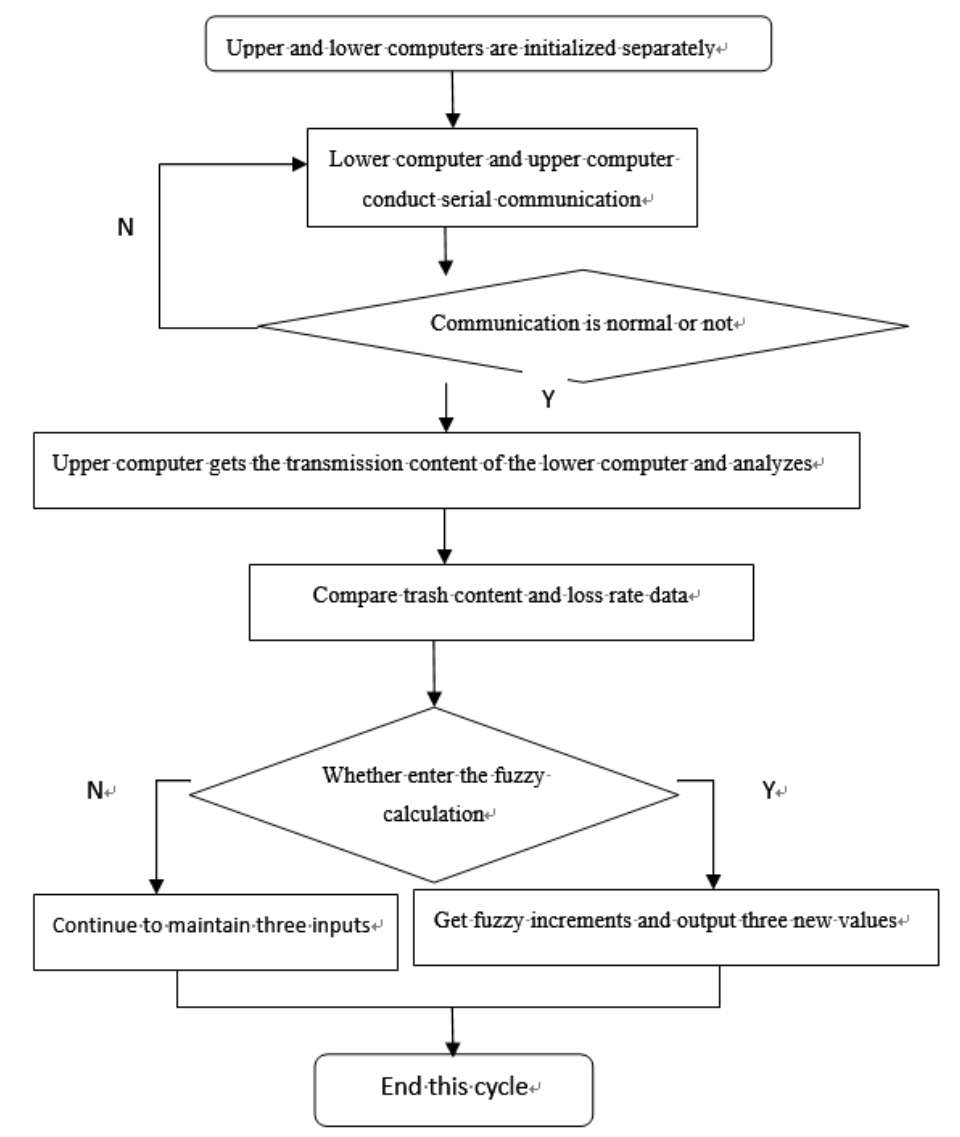

Figure 3. Flow chart of the monitoring system for cleaning

\section{Case-based Reasoning Cleaning Reasoning Intelligent Control Model}

\subsection{Theories and Applications of Case-based Reasoning}

Case-based reasoning was first proposed by Professor Schank of Yale University, he proposed in "Dynamic Memory: A Theory of Reminding and Learning in Computers and People" [7] in 1982, which is an important reasoning method in the field of artificial intelligence, and it is a simulation of human thinking and experience. When people are faced with a new problem, they often associate the previously used cases that are similar to the problem and use the experience and methods in the past to solve the current problems. Therefore, the core idea of this method lies in: when solving problems, solve the new problem by comparing the background and conditions of the new and old problems, or reusing or modifying the solution to the similar problems.

Based on the above methods and ideas, compared with other artificial intelligence systems, its advantages [8] are reflected in: CBR's knowledge acquisition is simply to obtain past cases, and does not require the establishment of explicit models; CBR system is applicable to incompletely formalized fields, incomplete areas of information and areas where experience and knowledge dominates, it is unnecessary for artificial intelligence (AI) experts and field experts to spend too much effort to write a lot of rules, CBR system can achieve self-learning by acquiring new cases and can handle large amounts of data.

CBR involves many fields in practical applications, such as fault diagnosis, decision support, medical diagnosis, and intelligent prediction and so on [9-18], through literature research, the casebased reasoning research results for the control strategy of rice and wheat combine harvester have not been reported. 


\subsection{Cleaning Intelligent Control Model}

In order to make the CBR method effectively and widely used, scholars built a model framework to achieve its versatility based on its core ideas. For example, Hunt [18] proposed the Hunt model based on the evaluation improvement strategy in 1995, Leaked [19] considered CBR as the process of "memory and application", expressed the application cycle of CBR as Ref, and proposed the CBR model with classification solution. In addition to the above two case-based reasoning models, most scholars, accept R4 cycle model constructed by Aamodt and Plaza [20], this model shows a better understanding for case-based reasoning thinking, and is more versatile in application; it has become the mainstream structure framework for case-based reasoning application system development. R4 divides the CBR process into four stages: retrieve (retrieve similar old cases), reuse (reuse solutions of old cases), revise (revise and adjust solution to old cases) and retain (retain new cases).

This paper consults the task flow in the R4 model, carries out the function module strategy design of cleaning recommendation system for the knowledge characteristics of cleaning control, and specifies the technologies applied in each task stage, solve the problems of knowledge representation, knowledge utilization and knowledge learning in the cleaning control scheme, The purpose is to implement the following functions: According to the attributes of the rice and wheat crop submitted by the users, it provides the most similar cleaning condition parameter plan in the normal range of cleaning mixing rate and cleaning loss rate. The principle of the work is as follows: first, the crop attributes data and the cleaning data of the grain production area are standardized and unified according to the hierarchy and framework of knowledge; and the case base of the cleaning control case is formed, then, the users can limit certain search conditions as the problem description part, and case-based reasoning system retrieves the cleaning control cases that match the current problem through similarity calculations, if the description parts of two problems are completely the same, then output cleaning control is output, otherwise, recommend cleaning control case with high similarity; users can recommend cleaning case to directly execute by the increase or decrease, or refer to the cleaning control cases formed by the attributes of similar crops, so form new cleaning control cases. Case; finally, feedback from the users decides the feasibility of the new case; then decides whether to join the case base as a new case, In order to solve the problem later. The system model is shown in Figure 4.

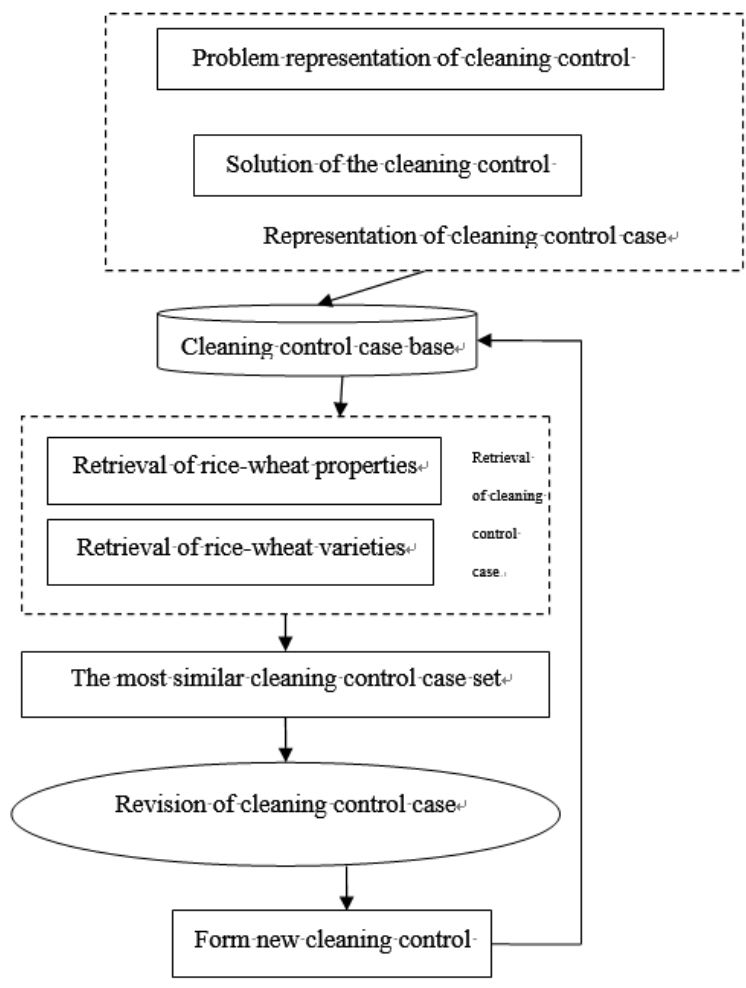

Figure 4. the flow chart of Case-based reasoning for cleaning 


\subsection{Case-based Reasoning Cleaning Intelligent Control Model}

This paragraph will study the important tasks such as representation, retrieval, and correction of cleaning control cases, and specifically describe how each task is implemented.

\subsubsection{Representation of Cleaning Case Control Models}

The case representation is the basis for the realization of case reasoning, in fact, the documents that used to solve the problems are represented in the form of cases and stored in the case base. The general case representation should include the problem description and the corresponding solution at least, namely, <problem description, solution> two-tuples form [16], which also clarifies the implementation part of case retrieval and case correction. The control strategy of the cleaning is to select the optimal cleaning control strategy based on moisture content and grain-straw ratio and crop varieties, achieve the minimum trash content and the loss rate. Therefore, crop properties such as moisture content and grain-straw ratio and crop varieties are problem descriptions, and an optimal cleaning control strategy is the solution. The following parameters can be used to express: moisture content $\mathrm{h}$, grain-straw ratio $\mathrm{c}$, crop variety $\mathrm{p}$; cleaning inclusion rate $\mathrm{z}$, cleaning trash content $\mathrm{s}$. draught distributing plate $\mathrm{f}$, fan speed $\mathrm{j}$, scale sieve opening $\mathrm{y}$. Therefore, the case which is composed of problem descriptions and solutions is represented as:

$$
\mathrm{C}_{\mathrm{k}}=\left(\mathrm{h}_{\mathrm{k}}, \mathrm{c}_{\mathrm{k}}, \mathrm{p}_{\mathrm{k}}, \mathrm{z}, \mathrm{s}: \mathrm{f}, \mathrm{j}, \mathrm{y}\right) \text {; }
$$

$\mathrm{k}$ is the number of historical cases in the case base. The parameters in the right part of formula (1) are described as problems; the parameters in the left part of formula (1) are used as the corresponding problem solution.

\subsubsection{Case Retrieval of Cleaning Control Model}

The retrieval of cases is mainly through the comparison of similarity to obtain the optimal solution; the accuracy of retrieval needs to rely on the calculation of similarity to a certain extent to be guaranteed. The definition of similarity is related to the quality of the retrieval results, if the definition is not clear, the retrieval result is not ideal, if it is over defined, it will lose the significance of artificial intelligence retrieval. For the case of cleaning control cases, there are mainly two kinds of characteristic attributes of the cases: semantic type and precise numerical types. Semantic type includes crop varieties, and precise numerical type includes moisture content and grain-straw ratio. The calculation method of similarity is different; the similarity calculation model of two kinds of attributes will be introduced later. The retrieval of the target case firstly judges whether the crop varieties in the case database correspond to each other, if they correspond, the similarity between the grain-straw ratio and the moisture content is calculated in the case matching case; if the crop varieties do not correspond, then similarity between grain-straw ratio and moisture content is calculated. Since grain-straw ratio and moisture content are very important factors in the performance of cleaning operations, therefore, this paper sets weight of the grain-straw ratio and the moisture content to 1 .

The similarity calculation between the cleaning grain-straw ratio and the cleaning water content was calculated with the Euclid distance. The calculation formula for the grain-straw ratio similarity is the square of the grain-straw ratio difference between the target cases in the case database; the calculation formula of the similarity of moisture content is the square of the difference between the target cases in the case database.

According to the above description, the target case is described as $\left(h^{\prime}, c^{\prime}, p^{\prime}, z^{\prime}\right)$. Its similarity and ith case $\mathrm{Ci}$ in the case base is defined as:

$$
\operatorname{Sim}\left(\mathrm{S}^{\prime}, \mathrm{Ci}\right)=\left(\mathrm{h}^{\prime}-\mathrm{hi}\right) 2+\left(\mathrm{c}^{\prime}-\mathrm{ci}\right)
$$

If the crop varieties match, select several cases as the most similar cases in the matching case set in accordance with the similarity, and return the results. If there is no matching case of crop variety, then select a few cases with the highest similarity and return the result.

\subsubsection{Cleaning Control Case Modification}

The modification of cleaning control cases is a key step in cleaning control case reasoning. Case retrieval was conducted based on varieties and attributes of crop, although this cleaning control case has a certain scientific characteristics, however, it is only a preliminary selection range for operators of the combined harvester based on the data from the historical case base, how to get the case that is most consistent with the setting parameters of field wheat and rice harvest condition according to 
similarity matching, the idea of the modification strategy of the cleaning control case is to conduct visual management for the retrieved cleaning control cases in this paper, specifically, the operator can set the number of retrieved cleaning cases, the retrieved selected cases, including crop varieties, crop attributes, cleaning condition parameters, cleaning trash content, and cleaning loss rate, are modified and perfected, make cleaning regulation case closer to actual situation, this can combine case retrieval and operators' experience, continuously optimize and enrich the construction of the cleaning control case base.

\subsubsection{Cleaning Control Case Storage}

Cleaning control case storage mainly achieves the storage of cleaning control strategy after it is revised, the stored case can cover existing cases; can also choose to store a new case. By adopting these two methods, it is possible to combine the operator's experience to revise the existing cleaning control cases, thus improving the case base system, can also target new rice varieties and properties, and add a new cleaning control strategy case associated with it.

\section{Management System of Cleaning Case}

The WEB-based cleaning control system adopts a common J2EE three-layer structure; the interface presentation layer adopts JSP tag technology and Extjs components to represent interface elements. The business logic part adopts EJB3.0 technology to encapsulate the business logic, and each different functional module is as a unit, they are packaged into EJB components, and the components can be freely combined. The data layer uses Hibernate + spring for data operations access to mysql.

Figure 5 is the rice-wheat varieties input interface of the cleaning case management system; the users can input rice and wheat varieties of the case through this interface.

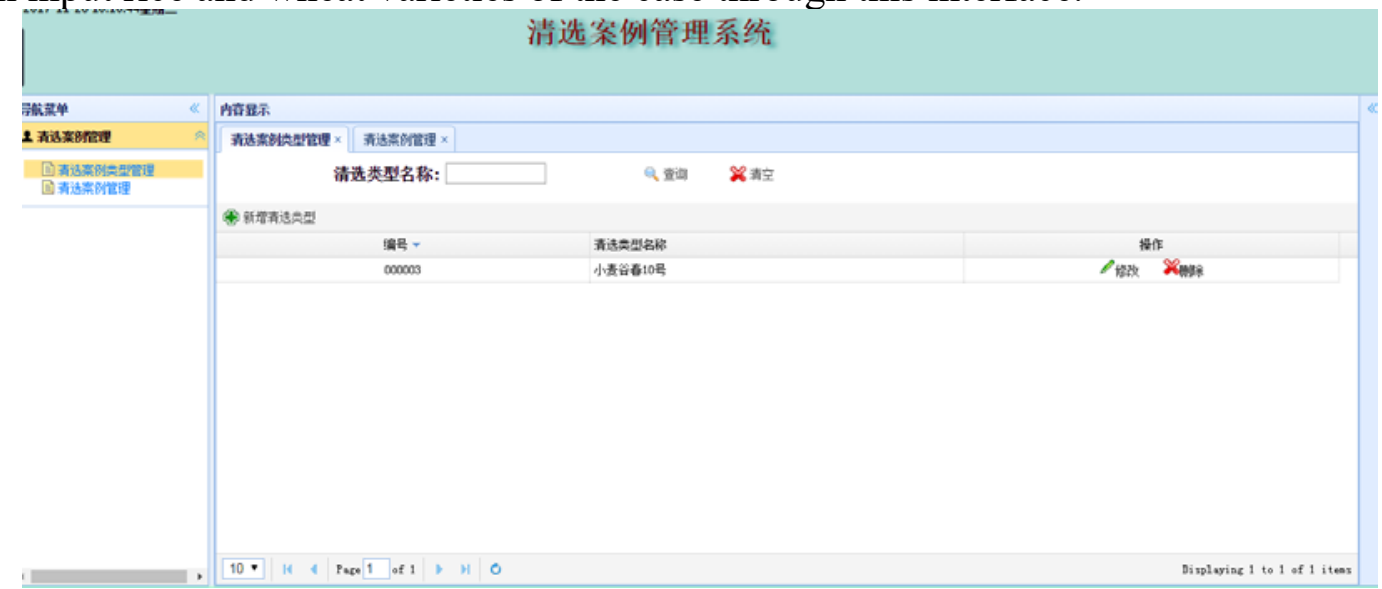

Figure 5. Input interface 1 of wheat and rice varieties

Figure 6 is the input interface of the cleaning case; the user selects the type entered in the figure above in "cleaning type name", as rice and wheat varieties in cases.

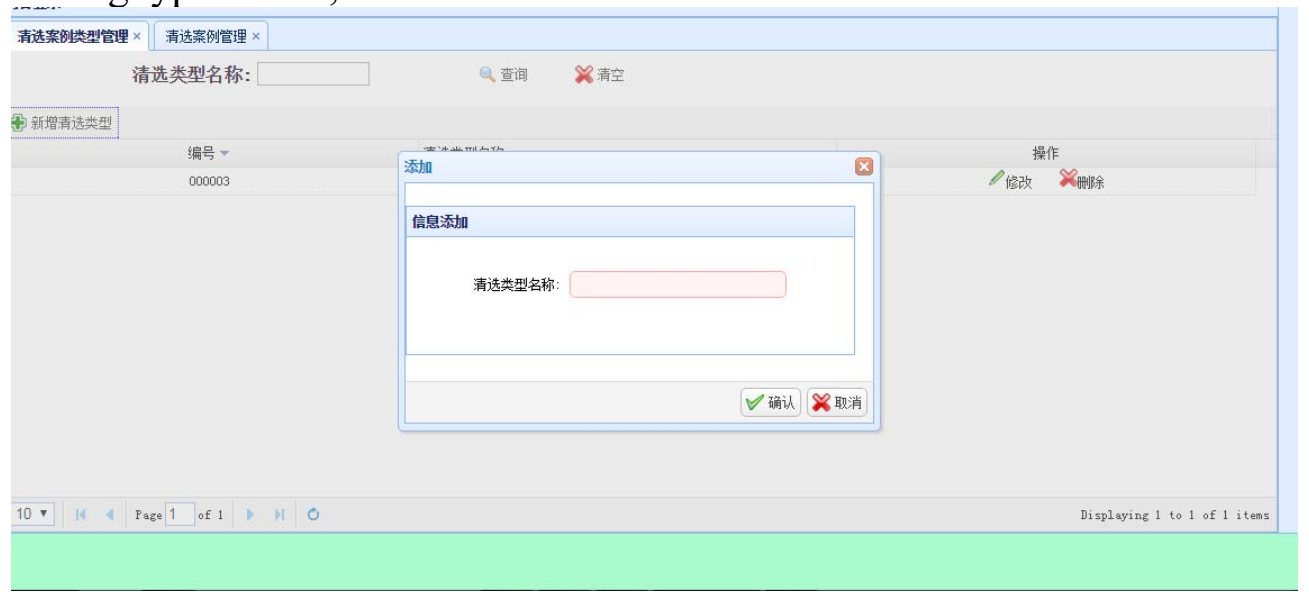

Figure 6. Cleaning interface of cleaning type 
Figure 7 is the input interface of the cleaning case, according to the input types in the figure, input the grain-straw ratio, moisture content and other rice-wheat properties, as well as the fan speed, draught distributing plate, and scale sieve opening and other cleaning parameters.

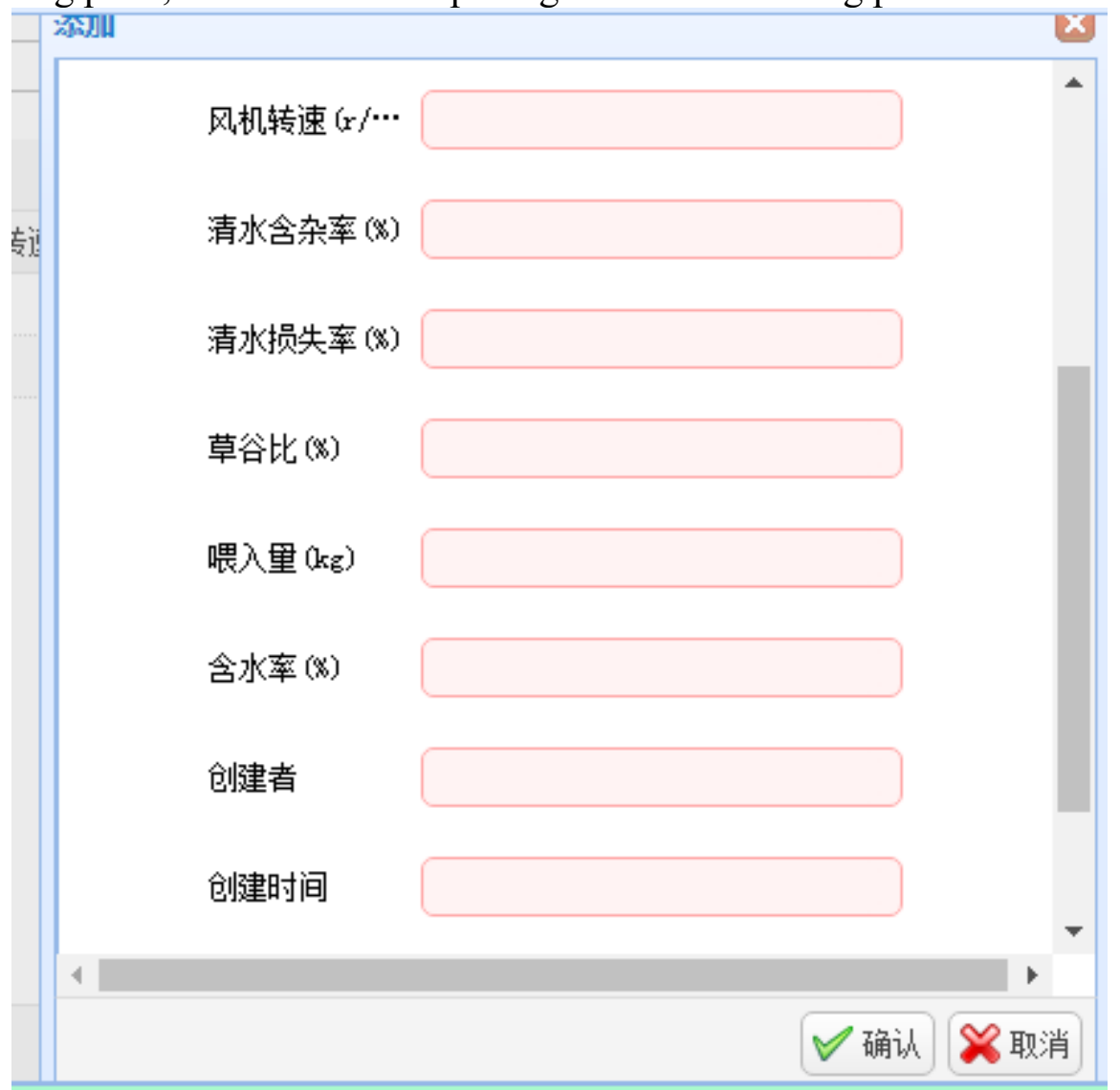

Figure 7. Cleaning Case Input Interface

Figure 8 is the management interface of the cleaning case system; any saved cleaning case can be opened, revised and saved.

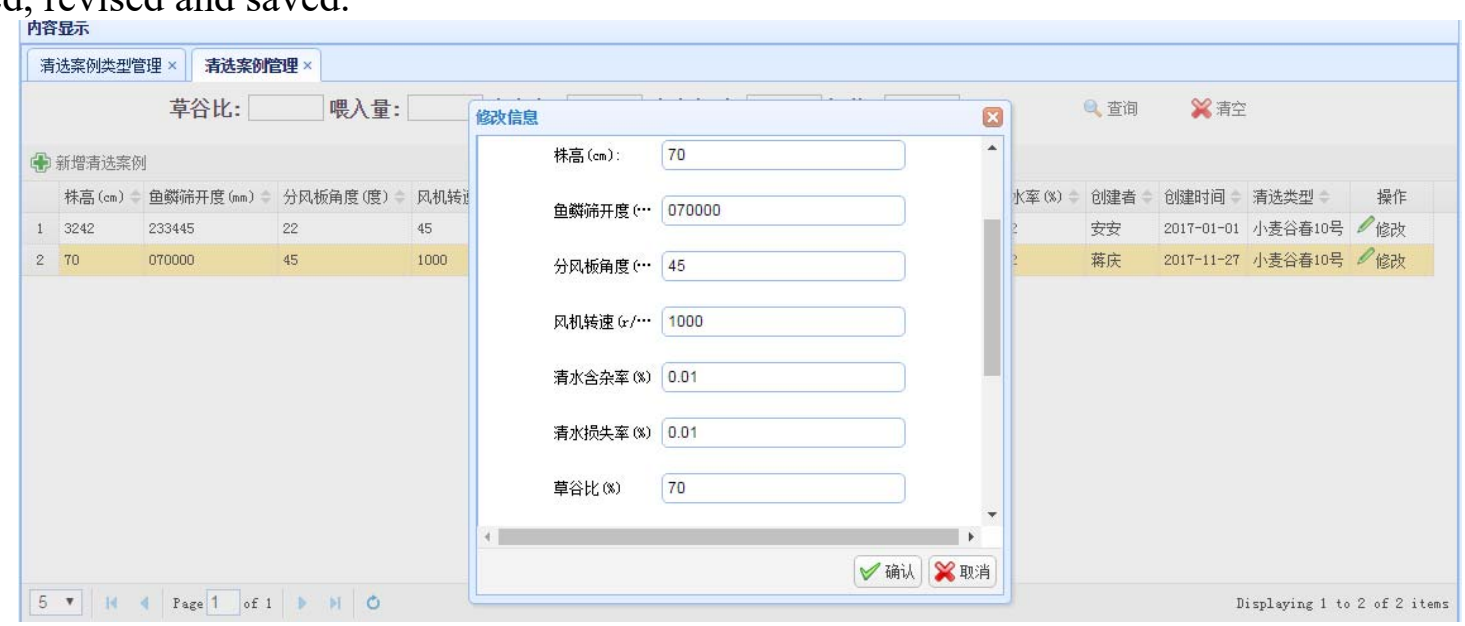

Figure 8. Editing Interface of Cleaning Case

Figure 9 is the main interface of the cleaning case retrieval; the user can input grain-straw ratio, feed quantity, moisture content, and other data to carry out related case queries, input data in the threshold, the system can display the case retrieval results whose similarity value is larger than the threshold in the main interface, it is convenient for users to analyze and edit the search results. 


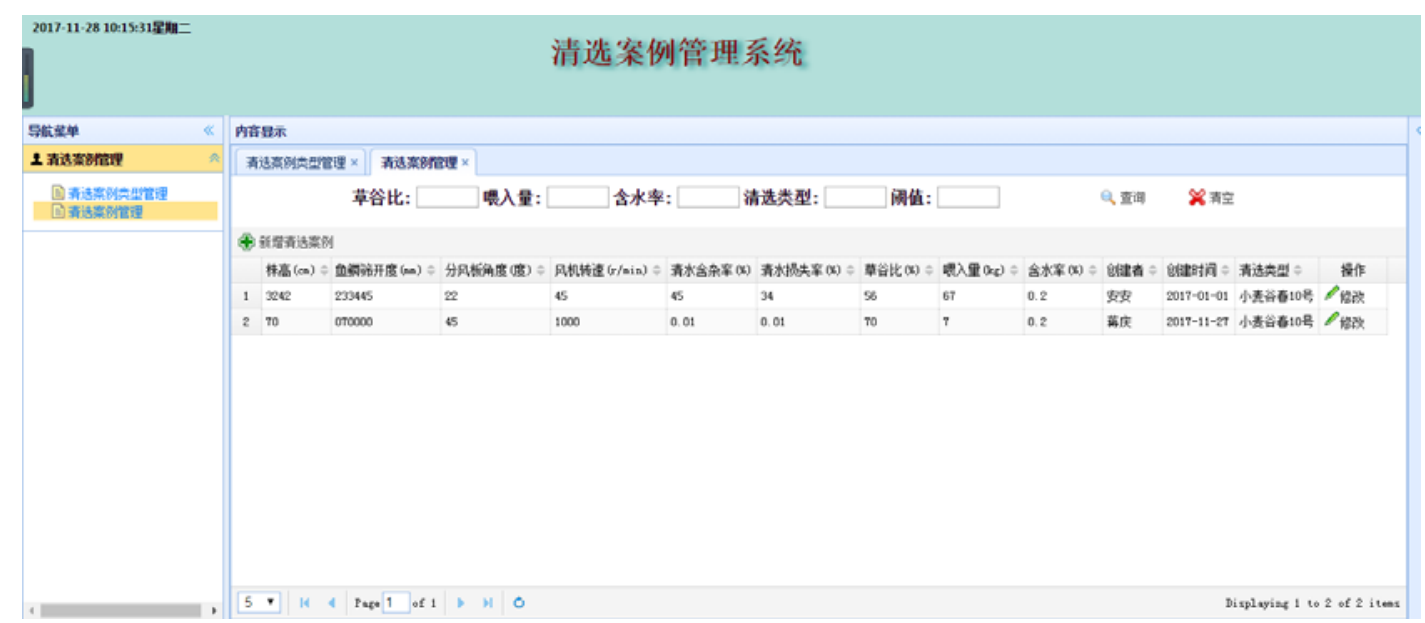

Figure 9. management main interface of cleaning case

\section{Conclusion}

This paper is aimed at the association relationship among the parameters of attributes and varieties of rice and wheat and the key parts of the rice and wheat combine harvester, the optimal trash content and loss ratios as goals, the cleaning control strategy case base, case retrieval, case modification, stored cleaning control model are established, the cleaning control case system for rice and wheat combine harvesters is developed based on Web technology, and valid the case-based reasoning to solve the effectiveness of the optimization problem of control parameters for the cleaning key components of the rice and wheat combine harvester.

\section{Acknowledgments}

This work was financially supported by the "Thirteenth Five-Year" National Key Research and Development Program of China No.2016YFD0702002, 2016YFD0702001, 2016YFD0702003, No.2017YFD0700705-3 and No.2017YFD0700501; And the National Natural Science Foundation of China under Grant No. 61503362, 61305111 and 91420104; Natural Science Foundation of Anhui Province under Grant No. 1508085MF133; Key technology and equipment research and development of intelligent harvest of Chinese wolfberry with high efficiency and low loss(No.nxzdkjxm2016-04); The National Natural Science Fund(No.31671586); Research and application of soil fertility rapid sensing device and large data fertilization model(15czz03129). The Research of Agricultural Science and Technology in Ningxia (No. XD-XMBW-2016001); Construction of test and maturation platform for complete sets of hardware and software in Agricultural Internet of things (Shanghai agriculture promotion word (2016) No. 2-5-11).

\section{References}

[1]. Gu Kejun, Xu Bo, Gu Dongxiang, Zhang Simei, Zhang Chuanhui, Shi Zi Liang, Yang Sijun, Straw/Grain Ratio of Wheat and Vertical Distribution of Wheat Straw in Jiangsu Province, Journal of Ecology and Rural Environment, 2015.

[2]. Xie Guanghui, Han Dongqian, Wang Xiaoyu, Lv Runhai, Harvest Index and Residue Factor of Cereal Crops in China, Journal of China Agricultural University, 2011.

[3]. Chen Du, Wang Shumao, Kang Feng, Zhu Qingyuan, Li Xiaohua, Mathematical Model of Feeding Rate and Processing Loss for Combine Harvester, Transactions of the Chinese Society of Agricultural Engineering, 2011.

[4]. Su Tiansheng, Study of Double Longitudinal Axial Flow Rice and Wheat Combine Harvester's Cleaning Device2015, Master's Thesis. 
[5]. Li Hongchang, Theoretical and Experimental Research on Wind Sieve Cleaning Device2011, $\mathrm{PhD}$ thesis.

[6]. Sun Guojian, Experimental Research on Air Sieve Double Air Channel Cross Flow Fan Cleaning Device, Master's Thesis.

[7]. Schank R C. Dynamic Memory: A Theory of Reminding and Learning in Computers and People[M] New York: Cambridge University Press, 1982.

[8]. Yang Tao. Research on Framework of Ontology Based Case-Based Reasoning System[D]. Nanjing: Nanjing University of Aeronautics and Astronautics, 2006.

[9]. Isabelle B. Case-base reasoning in the health sciences[J]. Artificial Intelligence in Medicine,2006,36(2):121-125.

[10]. Qu Gang, Tang Jiafu, et al. Adaptive Clinical Pathway System Based on CBR and MultiAgent [J] Systems engineering, 2012, 30 (11): 101-106.

[11]. Gao Shan, Zhu Yi, et al. Typhoon Case Reasoning Model Supported by GIS [J], Science of Surveying and Mapping, 2013, 38 (6): 46-48.

[12]. Shi Yonggui, Wang Hongfeng, et al. Case-based Decision Support System for Fault Management in CDMA Networks [J]. Journal of Northeastern University (Natural Science), 2011, 32 (7): 927-930943.

[13]. Lai yuan, Zhu Donghua, Guo Ying. Case-based Reasoning for Development of Enterprise's Patent Strategy [J].Science Of Science And Management Of S.\&.T., 2007, (8): 132-135.

[14]. Gao Xiaorong, Xu Yingzhuo, Li Qi. Intelligent Diagnosis and Process System Case-Based Reasoning for Drilling Complex Circs And Accident[J]. Applied Research of Computer, 2008, 25 (5): 1446-1449.

[15]. Liao Zhenliang, Liu Yanhui, Xu Zi Xin Case-Based Reasoning for Environmental Emergency Response and Preparedness System [J]. Environmental Pollution and Control, 2009, 31 (1): 8689.

[16]. Lv Zongping, Hu Xin, Yang Hongyu. Airport Emergency Preparednesss Management Based on Case-Based Reasoning [J]. Journal of Civil Aviation University of China, 2010, 28 (2): 1-5.

[17]. Li Meng, Liu Baoluo. Design on Mine Ventilation Fault Diagnoses Based on Case-Based Reasoning [J]. Coal Science and Technology, 2010, 38 (5): 9.

[18]. Hunt J. Evolutionary Case Based Design[A]. Waston I D. Progress in Case- Based Reasoning[M]. Berlin: Springer, 1995: 17-31.

[19]. Leake D B. Case - Based Reasoning: Experiences, Lessons and Future Direction[M]. Cambridge, MA, USA: MIT Press, 1996.

[20]. Aamodt A, Plaza E. Case-Based Reasoning: Foundational Issues, Methodological Variations, and System Approaches [J]. Artificial Intelligence Communications, 1996, 7 (1): 39 - 59. 